\title{
Coordinated Control of Networked Vehicles: An Autonomous Underwater System
}

\author{
F. Lobo Pereira and J. Borges de Sousa \\ Faculdade de Engenharia da Universidade do Porto, Porto, Portugal \\ Received October 28, 2003
}

\begin{abstract}
The specification and design of coordinated control strategies for networked vehicle systems are discussed. The discussion is illustrated with an example of the coordinated operation of two teams of autonomous underwater vehicles collecting data to find the local minimum of a given oceanographic scalar field.
\end{abstract}

\section{INTRODUCTION}

In this paper we discuss the problem of specification and design of coordinated control strategies for networked vehicle systems. This problem poses new challenges to control engineering. These challenges entail a shift in the focus of control engineering - from prescribing and commanding the behavior of isolated systems to prescribing and commanding the behavior of interacting systems. They can be accepted now due to dramatic technological developments in computing, communications, and control durind the last decade.

Today, and partly due to our involvement in the design and implementation of autonomous underwater vehicle systems for oceanographic and environmental field studies, we have a better understanding of the issues concerning the operation of multiple autonomous underwater vehicles [1]. In fact, these issues are quite general and are not specific to this type of vehicles.

In [2-4], we proposed a framework for the representation, formal specification, and control synthesis for networked vehicles. This framework is based on simple concepts from set theory and dynamic optimization. We use reach sets to describe the evolution of a dynamic system, invariant sets to describe the locations where the permanence of an entity within a certain set is ensured, and solvability sets to describe the locations from which a system can evolve to reach a given set. The key observation is that we can represent vehicles, their spatial-temporal evolution, their interactions, and their operations in the language of sets using concepts and techniques from dynamic optimization.

\section{PREVIEW OF THE OCEANOGRAPHIC MISSION}

Let us start with a preview of an oceanographic mission that can be a reality in a near future. Imagine two teams of AUVs that must coordinate their motions in order to find the local minimum of a scalar field pertinent to the description of a given oceanographic phenomenon.

The first team, denoted as LPS, provides a Local Positioning System (LPS) service to other team. This system can be viewed as an underwater version of the Global Positioning Service (GPS).

The second team, denoted as S, uses the LPS service for localization and provides a search service.

The Local Positioning System works as follows. Each LPS vehicle has a GPS receiver and an acoustic transponder - the vehicle is required to operate at the surface to receive the GPS signal 


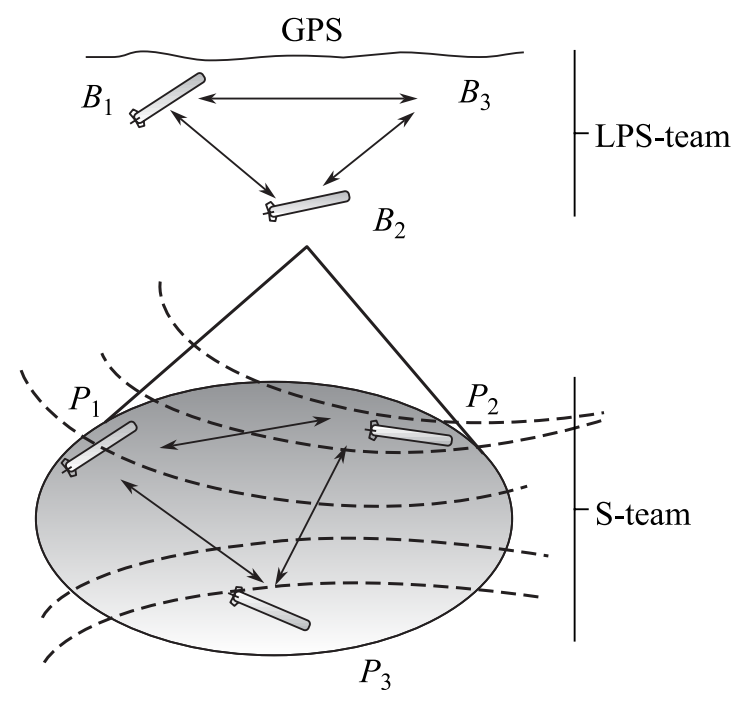

Figure.

providing time and position. The transponder emits regularly, with a known frequency, an acoustic ping encoding the name of the emitter as well as the time and the location of emission.

Each AUV from the S team is also equipped with an acoustic system. This system detects the arrival of acoustic pings, and decodes them to extract the position and name of the emitter and the time when the acoustic ping was emitted. This information together with the time of arrival of the ping is used to compute the distance between the AUV and the emitter. The calculation of the absolute position requires at least three LPS vehicles to form a simplex. Due to attenuation, the LPS service is only available within a neighborhood $P(t)$ of the LPS team.

The search for the local minimum is implemented as a modified version of the simplex optimization algorithm. Each S-vehicle has a suite of oceanographic sensors in addition to acoustic communication system.

In terms of motion coordination, the $\mathrm{S}$ team assumes the role of the leader. The LPS team controls the motions of its vehicles in order to keep all of the vehicles from $\mathrm{S}$ inside $P(t)$. The coordination results from information exchanged between the two teams.

\section{SPECIFICATION}

In order to design the coordinated control in the system under cosideration it is required a foemal description (specification of its elements, connections, services and constraints.

As a scalar oceanographic field the temperature field is considered. There are following sets of identical vehicles:

- $\mathcal{S V}$, of $n_{s}$ surface vehicles with a GPS receiver, a transponder, a radio, and an acoustic modem. The ranges of the radio, transponders, and acoustic modems are $r_{r}, r_{t}$ and $r_{a}$, respectively.

- $\mathcal{A U V} \mathcal{V}_{u}$, of $n_{u}$ AUVs with a Conductivity Temperature Depth $(C T D)$ sensor, an acoustic modem, and a navigation acoustic system. The range of the acoustic modem is $r_{a}$.

- $\mathcal{A U} \mathcal{V}_{s}$, of $n_{s u}$ AUVs mounting the same devices as the surface vehicles plus the sensor pack mounted on all $\mathcal{A U} \mathcal{V}_{u}$ vehicles. These multi-role vehicles may be assigned either to the LPS or to the $\mathrm{S}$ teams. 
At time $t$, the LPS team is composed of $n_{\mathrm{LPS}}(t)$ vehicles from $\mathcal{S} \mathcal{V}$ and from $\mathcal{A U \mathcal { V }}$, and the $\mathrm{S}$ team is composed of $n_{\mathrm{S}}(t)$ vehicles from $\mathcal{A U} \mathcal{V}_{u}$ and from $\mathcal{A U} \mathcal{V}_{s}$. We denote the $(x, y, z)$ position of the $j$ th vehicle from $S$ (LPS) by $X_{S, j}(t)\left(X_{\mathrm{LPS}, j}(t)\right), j=1, \ldots, n_{S}(t)\left(j=1, \ldots, n_{\mathrm{LPS}}(t)\right)$.

There are two distinct types of constraints: the ones required for a team to coordinate its operations and to maintain its integrity - the structural constraints, and the ones required for the team to provide services - service constraints. The structural constraints have precedence over the service constraints. The violation of the former imply the collapse of the team, while the violation of the latter degrades the way services are delivered.

The LPS vehicles must satisfy service and structural constraints.

Service constraints. The LPS team provides a positioning service to other vehicles. At time $t$, the service is available at all locations $X$ such that there are at least three LPS vehicles within distance $r_{t}$ - the range of the transponder - from $X$. The set of all such locations is denoted $P(t)$.

$$
\begin{gathered}
P(t)=\left\{X \in \Re^{3}: \exists i, j, k \in \mathrm{LPS}, \quad k \neq i \wedge i \neq j \wedge j \neq k \wedge d\left(X_{\mathrm{LPS}, j}, X\right)\right. \\
\left.\leq r_{t} \wedge d\left(X_{\mathrm{LPS}, i}, X\right) \leq r_{t} \wedge d\left(X_{\mathrm{LPS}, k}, X\right) \leq r_{t}\right\}, \quad d(X, Y)=\|X-Y\| .
\end{gathered}
$$

At least three vehicles from LPS are required to form a simplex. We express a relaxed version of this requirement using the distance function $d$ as follows:

$$
\begin{gathered}
\exists i, j, k \in \mathrm{LPS} \quad \text { with } \quad j \neq i, i \neq k, k \neq j \quad \text { such that: } \\
d\left(X_{\mathrm{LPS}, i}, X_{\mathrm{LPS}, k}\right)<d\left(X_{\mathrm{LPS}, i}, X_{\mathrm{LPS}, j}\right)+d\left(X_{\mathrm{LPS}, j}, X_{\mathrm{LPS}, k}\right),
\end{gathered}
$$

where $d(X, Y)=\|X-Y\|$ is the distance function.

Structural constraints. The LPS vehicles have to coordinate their motions to satisfy the service constraints, and to follow the $\mathrm{S}$ team. This is why we require the LPS vehicles to form a communication network where every two distinct vehicles should be able to communicate between them. We express the requirement as graph connectedness. To express graph connectedness formally we need some terminology and notions from graph theory. Define the graph $T$ as follows. Each vehicle in LPS is represented by a vertex. There is an edge between two vertices whenever the distance between the corresponding vehicles is less than the radio communication range $r_{r}$. The communication constraints are expressed as follows: the graph $T$ is connected.

The S team vehicles implement a modified version of the simplex algorithm. The implementation of this algorithm requires permanent communication among the vehicles. Here, again, we express the communication constraint as the connectedness of the graph $K$ defined as follows. To each vertex in $K$ there corresponds a vehicle in S. There is an edge between two vertices whenever the distance between the corresponding vehicles is less than $r_{a}$.

LPS-S coordination require the folloving two constraints:

(1) the vehicles from the $\mathrm{S}$ team should remain inside the set $P(t)$, i.e.,

$$
X_{S, i}(t) \in P(t), \quad i=1, \ldots, n_{S}(t),
$$

(2) the vehicles in both teams should be able to communicate among themselves. If $r_{a}$ is the maximum range of communication we express this constraint as follows:

$$
\min _{i, j} d\left(X_{\mathrm{LPS}, j}(t), X_{S, i}(t)\right) \leq r_{a} \quad \forall t
$$

Consider, for the sake of simplicity, a scalar field $q(x): \Re^{2} \rightarrow \Re$ evolving in the horizontal plane with a unique local minimum in the region of interest. We are interested in finding this minimum. The $3 \mathrm{D}$ extension of this problem is straightforward. 
At the beginning of each iteration $i$, we have three points $A_{i}, B_{i}, C_{i}$, and the corresponding values of the scalar field $q\left(A_{i}\right), q\left(B_{i}\right), q\left(C_{i}\right)$. We have labelled the points so that $q\left(A_{i}\right) \leq q\left(B_{i}\right) \leq q\left(C_{i}\right)$. The sequence of computations is described next.

Algorithm. Take $i:=1$. Consider the points $A_{1}, B_{1}, C_{1}$ forming a triangle. Repeat until finding minimum.

(1) Take the segment joining $A_{i}$ and $B_{i}$. Define the midpoint of this segment as $z_{i}$. Define the cone $K\left(z_{i}\right)$ with apex at $z_{i}$ of all the unit vectors from $C_{i}$ to $z_{i}+\delta B$, where $\delta$ is a parameter and $B$ the unit ball.

(2) Define the set of feasible directions at $z_{i}$ as $K\left(z_{i}\right)$.

(3) Select one vector $v$ from $K\left(z_{i}\right)$.

(4) If, at any point $z_{i}+\delta B$, and for any direction $w$ such that $\langle w, v\rangle \geq 0$, the directional derivative of $f$ along $w$ is non-negative, i.e., $f^{\prime}\left(z_{i} ; w\right) \geq 0$, then stop. In this situation, the point of minimum is located within the triangle with vertices $A_{i}, B_{i}$, and $C_{i}$. Otherwise, find the minimum of $\mathrm{f}$ in the direction of $v$ starting at $z_{i}$. Denote the point where the minimum is attained as $A_{i+1}$. Then $f\left(A_{i+1}\right) \leq f\left(A_{i}\right) \leq f\left(B_{i}\right) \leq f\left(C_{i}\right)$.

(5) Rename $A_{i}$ and $B_{i}$ as $B_{i+1}$ and $C_{i+1}$ respectively.

(6) Set $i:=i+1$.

This algorithm is scalable with respect to the number of vehicles used to implement it. With one vehicle, the ideal implementation would require this vehicle to jump from point $A_{i+1}$ to point $z_{i+1}$ at the beginning at each new iteration. With two vehicles, the ideal implementation would require one of them to be at position $z_{i+1}$ when the other reaches the point $A_{i+1}$.

\section{COORDINATION AND CONTROL}

In this section we discuss the control and coordination problems arising in this mission specification. We show how the specification can be mapped onto control formulations within the framework of dynamic optimization [1-13], and how the intra and the inter team coordination problems can be formulated as nested problems of invariance. Finally, we briefly discuss the conceptual solution methodology.

The $\mathrm{S}$ team plays the role of the leader in the coordinated operation of both teams. The leading role stems from the implementation of the modified simplex algorithm. The connectedness of graphs $K$ and $T$ and condition (3.2) represent invariance requirements for the vehicles from both teams. Likewise, the coordination conditions (3.3) and (3.4) represent an invariance requirement for the LPS team.

\subsection{Implementation of the Modified Simplex Algorithm}

A scalable implementation of this algorithm requires two basic motion patterns for each iteration. One, called the leader pattern, requires one vehicle, the leader, to enter the cone of descent directions $K\left(z_{i}\right)$ at the apex $z_{i}$, and to move in one of those directions with maximum speed until a maximum is found. The other, let us call it the competition for leadership pattern, requires the other vehicles to compete to become the leader in the next iteration. The competition consists in finding the vehicle that will reach in minimum time the apex $z_{i+1}$ of the cone $K\left(z_{i+1}\right)$ of descent directions for iteration $i+1$. This is simultaneously a tracking and a reachability problem with state constraints. Let us assume that, at each time $t \geq t_{i+1}$, the position of the leader is known, and let us call it $\bar{A}_{i+1}$. Define $\bar{z}_{i+1}$ and $\bar{K}\left(\bar{z}_{i+1}\right)$ as before. The objective for each vehicle, with the exception of the current leader, is to move with respect to cone $\bar{K}\left(\bar{z}_{i+1}\right)$ with apex $\bar{z}_{i+1}$ in 
such a way that it can become the leader in the next competition. Let $T_{k}^{*}(i)$ be the minimum time required for vehicle $k$ to reach a ball $\mathrm{B}$ of radius $\delta$ centered on $z_{i}$ - the midpoint between $A_{i}$ and $B_{i}$ and the apex of cone $K\left(z_{i}\right)$-from its current position $X_{S, k}\left(t_{i}\right)$. Then, the leader for the next iteration is given by:

$$
\arg \min _{k}\left\{T_{k}^{*}(i): X_{S, k}\left(T_{k}^{*}(i)\right) \in B_{\delta}\left(z_{i}\right)\right\} .
$$

Actually we want the vehicle to penetrate the cone. Hence, we have to add the following constraint to the previous minimization: $\dot{X}_{S, k}\left(T_{k}^{*}(i)\right) \in K\left(z_{i}\right)-\left\{z_{i}\right\}$.

\subsection{Inter-Team Motion Coordination}

The coordination and control problem for the LPS team consists in finding a controller such that the following constraints are satisfied simultaneously: (1) service constraints (given by Eq. (3.2)), (2) structural constraints (connectedness of the graph $T$ ), and (3) coordination constraints (given by Eqs. (3.4) and (3.3)).

This is basically a problem of controlled invariance, where the LPS team controls the motions and shape of the set $P(t)$. There are at least three basic formulations for this coordination and control problem: (1) constraining the motions of the LPS vehicles; (2) constraining the motions of the S vehicles; (3) constraining both the motions of the $\mathrm{S}$ and LPS vehicles. The last formulation requires a two-way information flow between the two teams. This comes for free in our example. The first two formulations assume that one the teams is able to solve the control and coordination problem. But it may not be possible to leave all the coordination burden to one of the teams.

In the next section we formulate this problem under the following assumptions: (1) the state of the $\mathrm{S}$ team is known and, (2) S does not cooperate with LPS, i.e., the coordination and control burden is placed on the vehicles of LPS team. Hence, the $\mathrm{S}$ team broadcasts its state to make it available to the LPS team. This is feasible as far as the communication condition (3.4) is satisfied. At each time $t$, with $t_{i}<t<t_{i+1}$, the state of $\mathrm{S}$ is described by $z_{i}$ and $K\left(z_{i}\right), \overline{A_{i+1}}$ and $\bar{K}\left(\bar{z}_{i+1}\right)$, $n_{S}(t)$, and $X_{S, i}(t)$ for all vehicles in $\mathrm{S}$.

\subsection{Intra and Inter-Team Invariance}

LPS intra-team invariance. The dynamics of each vehicle $i$ is given by the following equation:

$$
\begin{aligned}
\dot{x}_{\mathrm{LPS}, i}(t) & =f_{\mathrm{LPS}, i}\left(x_{\mathrm{LPS}, i}(t), u_{\mathrm{LPS}, i}(t)\right), \\
u_{\mathrm{LPS}, i}(t) & \in U_{\mathrm{LPS}, i}(t), \\
x_{\mathrm{LPS}, i}(t) & \in \Re^{k} .
\end{aligned}
$$

The function $f_{\mathrm{LPS}, i}$ specified the general motion equations of the $i$ th vehicle of the LPS team which are given by (see [14] or [15]),

$$
\begin{gathered}
\dot{\eta}=J(\eta) \nu \\
M \dot{\nu}+C(\nu) \nu+D(\nu) \nu+g(\eta)=\tau,
\end{gathered}
$$

where $\eta:=\operatorname{col}(x, y, z, \phi, \theta, \psi)$ is the position and orientation in the external navigation frame, and $\nu:=\operatorname{col}(u, v, w, p, q, r)$ is the linear and angular velocities in the body frame. Here, $M$ is the inertial matrix which includes the effects of the added mass (fluid displacement inertia) of the vehicle, $C$ is the centripetal and Coriolis matrix, $D$ is the matrix corresponding to hydrodynamic lift and drag forces/moments, $J$ is a transformation matrix, $g$ is the restoring forces and moments caused by gravity and buoyancy, and $\tau$ is the external forces and moments. 
The team must satisfy two types of state constraints: service constraints; and structural constraints. These constraints are mapped onto the disjunction of $j=1, \ldots, J$ sets of state constraints in $X=\Re^{k \times n_{\mathrm{LPS}}(t)}$. Each set of state constraints is obtained from the conjunction of service and structural constraints and is expressed as follows:

$$
\varphi_{j}\left(t, x_{\mathrm{LPS}}(t)\right) \leq 1, \quad \varphi_{j}^{0}\left(x_{\mathrm{LPS}}(0)\right) \leq 1, \quad j=1, \ldots, J .
$$

For $i=1, \ldots, n_{\text {LPS }}$, denote by $\bar{x}_{\text {LPS }}^{i}$ the component of $x_{\text {LPS }}^{i}$ corresponding to the position of the $i$ th LPS vehicle in $\Re^{3}$ and let $R$ be the communication range. Then, an instance of the above function in order to ensure communication among all vehicles could be

$$
\varphi_{i}\left(t, x_{\mathrm{LPS}}\right)=\max \left\{\frac{d\left(\bar{x}_{\mathrm{LPS}}^{i}, \bar{x}_{\mathrm{LPS}}^{k}\right)}{R}: k=1, \ldots n_{\mathrm{LPS}}, k \neq i\right\} .
$$

Another function to ensure the localization service is delivered for some region of the ocean is given by

$$
\max \left\{\frac{d\left(\bar{x}_{\mathrm{LPS}}^{i}, \bar{x}_{\mathrm{LPS}}^{k}\right)+\varepsilon_{\mathrm{LPS}}}{d\left(\bar{x}_{\mathrm{LPS}}^{i}, \bar{x}_{\mathrm{LPS}}^{j}+d\left(\bar{x}_{\mathrm{LPS}}^{j}, \bar{x}_{\mathrm{LPS}}^{k}\right)\right.}: i, j, k \text { s.t. } \bar{x}_{\mathrm{LPS}}^{i} \neq \bar{x}_{\mathrm{LPS}}^{j}, \bar{x}_{\mathrm{LPS}}^{j} \neq \bar{x}_{\mathrm{LPS}}^{k}\right\},
$$

where $\varepsilon_{\text {LPS }}$ is some constant chosen in order to ensure a pre-specified maximum worst case localization error.

This is a problem of weak invariance since we are interested in the existence of at least one control law ${ }^{1}$. The general problem formulation is the following:

Problem 4.1. Find the largest invariant set $\mathcal{W I}_{\mathrm{LPS}}(t)$ such that, if the initial position of the system lies in this set, it is always possible to find a control $u_{\mathrm{LPS}}(t)$ that prevents the state of the system to violate condition (4.8).

Following the arguments from [13], we can prove that the set $\mathcal{W} \mathcal{I}_{\mathrm{LPS}}(t)$ is given by the level sets of a suitable value function. In order to do this let us first introduce some notation:

$$
\phi(\tau, x):=\max \left\{\varphi_{k}(t, x)\right\} \quad \phi^{0}(x):=\max \left\{\varphi_{k}^{0}(x)\right\} .
$$

Consider the following value function:

$$
\left.V(\tau, z)=\min _{u_{\mathrm{LPS}}\left(t_{0}, \tau\right)}\left\{\min \left\{\phi\left(\tau, x_{\mathrm{LPS}}\right), \phi^{0}\left(x_{\mathrm{LPS}}\left(t_{0}\right)\right)\right\} \mid x_{\mathrm{LPS}}(\tau)=z\right)\right\} .
$$

Let $\Pi(z)$ be the projection of $z$ on $3 n_{\mathrm{LPS}}$ - dimensional space of pozitions. Now we can easily see that:

$$
\mathcal{W I}_{\mathrm{LPS}}(t)=\Pi(\{z: V(t, z) \leq 1\})
$$

The patterns of motion for the LPS vehicles are constrained to belong to this set. Similar results for strong invariance are obtained if the minimization in equation (4.10) is replaced by a maximization.

The question now is how to calculate the value function. This is not a trivial matter. By using classic arguments from [16], we can prove that this value function satisfies the principle of

\footnotetext{
${ }^{1}$ In our illustrative oceanographic mission we are interested in constraining just the $(x, y, z)$ components of the state of all vehicles.
} 
optimality. In this case the value function $V$ can be calculated by solving the generalized HamiltonJacobi-Bellman (HJB) equation. In fact, an infinitesimal version of the principle of optimality leads to Hamilton-Jacobi-Bellman equation:

$$
\begin{gathered}
V_{t}(t, x)+\max _{u \in U_{\mathrm{LPS}}(t)}\left\{V_{x}(t, x) \times f_{\mathrm{LPS}}(t, x, u)\right\}=0, \\
V\left(t_{0}, x\right)=\phi^{0}(x),
\end{gathered}
$$

where $V_{t}, V_{x}$ represent the corresponding subdifferentials. This results from the fact that the value function is generally nondifferentiable. The usual notion of solution of a partial differential equation does not apply in this case since $\mathrm{V}$ is nondifferentiable. We consider generalized "viscosity," or equivalent concepts, of solutions for this equation (see [16-19]).

The characterization of the value function as a solution of the Hamilton-Jacobi-Bellman equation is at the heart of the Dynamic Programming approach to solve dynamic optimization problems for which several numerical methods (see [16] and references therein) are available. Although, this approach suffers from the "curse of dimensionality" for general control problems, it is well known that, for problemas with quadratic cost and linear dynamics, it reduces to solving a differential Riccati equation.

The motions of the $\mathrm{S}$ team vehicles and the conditions of $\mathrm{S}$ intra-team invariance are described analogously. The corresponding invariant set is $\mathcal{W I}_{S}(t)$.

Inter-team invariance. At each time $t$, each vehicle $i$ of $\mathrm{S}$ is required to remain inside $P(t)$, i.e.,

$$
\forall i, t \quad x_{S, i}(t) \in P(t)
$$

We represent this constraint with a set-valued map $J$ mapping the state of LPS onto a subset of $\Re^{3}$, i.e., $P(t)=J\left(x_{\mathrm{LPS}}(t)\right)$.

In order to write this inter-team state constraint in the form of an inequality such as (4.9) we consider the following "distance" function $d_{A}(B)$ of the closed set $B$ to the closed set $A$, both in $\Re^{k}$ :

$$
d_{A}(B):=\max \left\{d_{A}(x): x \in B\right\},
$$

where $d_{A}(x)$ is the Euclidean distance between the point $x$ and the set $A$. Now define:

$$
\varphi_{\mathrm{LPS}}\left(t, x_{\mathrm{LPS}}(t)\right)=d_{\mathcal{W I}_{S}(t)}\left(J\left(x_{\mathrm{LPS}}(t)\right)\right)+1 .
$$

It is now possible to express this additional constraint on the motions of the LPS vehicles as follows:

$$
\varphi_{\mathrm{LPS}}\left(t, x_{\mathrm{LPS}}(t)\right) \leq 1
$$

In practice, the LPS team defines a state constraint for all the vehicles in S. But, according to the previous section, we want the $\mathrm{S}$ vehicles to satisfy this constraint without additional control effort from the elements of this team. Hence, we want to solve the following problem:

Problem 4.2. Find the largest invariant set $\mathcal{W I}_{\mathrm{LPS}_{S}}(t)$ such that if the initial positions of the LPS vehicles lie in this set, it is always possible to find controls $u_{\mathrm{LPS}}(t)$ and $u_{S}(t)$ such that condition (4.16) is never violated.

See [5] for a discussion on how to solve this problem. 


\section{CONCLUSION}

We can find the same patterns of interactions in other problem domains, for example in military, scientific and civilian applications involving Unmanned Air Vehicles (UAV)s or Unmanned Combat Air Vehicles (UCAV) (see [20] for an extensive survey on applications of these vehicles). Military operations present the most challenging scenarios. These include reconnoitering areas that may be contaminated, surveillance, zone interdiction or sentry duty, and suppression of enemy air defenses (SEAD). In SEAD missions we have spatial and temporal rendezvous where vehicles form teams. In zone interdiction missions we have teams of UCAVs that coordinate their motions to maximize the area covered by unit of time. In reconnaissance and target finding missions we have search-based algorithms with integration of data from different sensors mounted on different vehicles.

From the analysis of the oceanographic mission we may infer the specificity of such applications, which require to coordinate the motion and functioning of heterogeneous vehicles.

It is generally the case that space is at premium in autonomous vehicles, whether for sea, air, or land applications. Moreover, sensing and sampling strategies may require the spatial distribution not only of sensors, but also of components of the same sensing system. In both cases we need to distribute capabilities - multiple sensors or different components of the same sensor - among different vehicles.

Some services, for example communication services, have to be distributed among multiple vehicles to cover a given area.

At a certain level of abstraction, vehicles are points in the 3D space. The motion requirements for several applications, for example in the oceanographic mission, are expressed as an algorithm that may, or may not, be implementable with the motions of those points.

The teams are designed to provide services that cannot be delivered by a single vehicle. This means that vehicles within a team and teams within interacting teams have to coordinate their activities to provide services. This is done according to well-defined patterns of coordination and control. For example, the vehicles in our oceanographic mission exhibit patterns of coordination and control which are quite general.

The delivery of a service requires the satisfaction of predicates on the capabilities and on the relative motions of the vehicles providing the service.

A team comes into existence through the coordinated activities of its vehicles. This means that, with respect to other teams, each team acts as a single entity, thus engaging, as an unit, in interactions with those teams. For example, the LPS follows the $\mathrm{S}$ team in order to keep the vehicles from $\mathrm{S}$ within $P(t)$.

Services may build on other services. In fact, we may need to recruit the utilization of several teams to deliver a service. This requires the nesting of services, of constraints, and of controllers. For example, the LPS and the S team jointly provide a search service. The modes of coordination at this level are richer than the modes of coordination at the intra-team level. First, teams form spatial entities whose shape and evolution we may want to control. Second, it may be possible, and desirable, to transfer assets among teams.

In order to coordinate their activities, the vehicles within a team, and groups of teams, interact among themselves and with other entities. To do this, they establish and destroy links among them. This means that they form a system with an evolving structure. Hence, establishing or destroying a link is a control action that may result in a different behavior for the structure.

In the whole the approach proposed presumes formal specification which uses logical statements and set-theoretic constructs that are amenable to mathematical manipulation at the design stage.

We define invariants, that we require the implementation to satisfy. The invariant captures the essence of what makes an implementation correct. 
In what concerns design, we formulate the coordination and control problems in the setting of dynamic optimization. In this setting we express complex requirements, such as the disjunction of joint-state constraints and relative motion coordination, in terms of invariance of level sets of value functions, and of reachability. In doing this, we are able to derive conditions under which the invariants will be true, and synthesize controllers that ensure invariance. In summary, the design is a refinement of the specification.

\section{ACKNOWLEDGMENTS}

Research supported in part by Fundação da Ciência e Tecnologia project Cordyal, by Fundação das Universidades Portuguesas project COOP, and by the Luso-American Development Foundation Fellowships program.

\section{REFERENCES}

1. de Sousa, J.B. and Sengupta, R., Cdc Tutorial on Autonomous and Semiautonoomous Networked Multivehicle Systems, 2001.

2. Pereira, F.L., Control Design for Autonomous Vehicles: A Dynamic Optimization Perspective, Eur. J. Control, 2001, no. 7, pp. 178-202.

3. Pereira, F.L. and de Sousa, J.B., Specification and Design of Coordinated Motions for Autonomous Vehicles, IEEE Conf. on Decision and Control Society, 2002.

4. Pereira, F.L., de Sousa, J.B., and Matos, A., Dynamic Optimization in the Coordination and Control of Autonomous Underwater Vehicles, IEEE Conf. on Decision and Control, 2002.

5. Aubin, J.-P., Viability Theory, Boston: Birkhauser, 1991.

6. Clarke, F.N. et al., Nonsmooth Analisis and Control Theory, New York: Springer, 1998.

7. Clarke, F.H., Optimization and Nonsmoth Analisis, Philadelphia: SIAM, 1990.

8. Clarke, F.H., A Proximal Characterization of the Reachable Set, Syst. Control Lett., 1996, vol. 27, no. 3, pp. 195-197.

9. Clarke, F.H. and Wolenski, P.R., Control of Systems to Sets and Their Interiors, J. Optimiz. Theory Appl., 1996, vol. 88, no. 1, pp. 3-23.

10. Clarke, F.H., Ledyaev, Y.S., and Subbotin, A.I., The Synthesis of Universal Feedback Pursuit Strategies in Differential Games, SIAM J. Control Optimiz., 1997, vol. 35, no. 2, pp. 52-61.

11. Krasovskii, A.N., Control under Lack of Information, Boston: Birkhauser, 1995.

12. Advances in Nonlinear Dynamics and Control: A Report from Russia, Kurzhanskii, A.B., Ed., Boston: Birkhauser, 1993.

13. Kurzhanskii, A.B., Ellipsoidal Calculus for Estimation and Control, Boston: Birkhauser, 1997.

14. Fossen, T.I., Guidance and Control of Ocean Vehicles, New York: Wiley, 1994.

15. Principles of Naval Architecture, Lewis, E., Ed., Society of Naval Architects and Marine Engineers, 1989.

16. Bardi, M. and I. Capuzzo-Dolcetta, I., Optimal Control and Viscosity Solutions of Hamilton-JacobiBellman Equations, Boston: Birkhauser, 1997.

17. Evans, L.C., Partial Differential Equations. Graduate Studies in Mathematics, Providence: AMS, 1998.

18. Krasovskii, N.N. and Subbotin, A.I., Game-theoretical Control Problems, New York: Springer-Verlag, 1988.

19. Subbotin, A.I., Generalized Solutions of First-order PDEs: The Dynamical Optimization Perspective, Boston: Birkhauser, 1995.

20. Van Cleave, D., Trends and Technologies for Uninhabited Autonomous Vehicles, in Software-Enabled Control: Information Technology for Dinamical Systems, Samad, T. and Balas. G., Eds., New York: Wiley, 2002.

This paper was recommended for publication by V.N. Bukov, a member of the Editorial Board 